\title{
Use of the Clock Drawing Test and the Rey- Osterrieth Complex Figure Test-copy With Convolutional Neural Networks to Predict Cognitive Impairment
}

\section{Young Chul Youn}

Chung-Ang University Hospital https://orcid.org/0000-0002-2742-1759

Jung-Min Pyun

Seoul National University Bundang Hospital

Nayoung Ryu

Seoul National University Bundang Hospital

Min Jae Baek

Seoul National University Bundang Hospital

Jae-Won Jang

Kangwon National University School of Medicine

Young Ho Park

Seoul National University Bundang Hospital

\section{Suk-Won Ahn}

Chung-Ang University Hospital

\section{Hae-Won Shin}

Chung-Ang University Hospital

\section{Kwang-Yeol Park}

Chung-Ang University Hospital

\section{SangYun Kim ( $\nabla$ neuroksy@snu.ac.kr)}

Seoul National University College of Medicine \& Neurocognitive Behavior Center, Seoul National University Bundang Hospital https://orcid.org/0000-0002-9101-5704

\section{Research}

Keywords: Convolutional neural network, Clock Drawing Test, Rey-Osterrieth Complex Figure Test, Cognitive impairment, Machine learning, TensorFlow

Posted Date: December 15th, 2020

DOl: https://doi.org/10.21203/rs.3.rs-125100/v1 
License: (c) (i) This work is licensed under a Creative Commons Attribution 4.0 International License. Read Full License

Version of Record: A version of this preprint was published at Alzheimer's Research and Therapy on April 20th, 2021. See the published version at https://doi.org/10.1186/s13195-021-00821-8. 


\section{Abstract}

Background: The Clock Drawing Test (CDT) and Rey-Osterrieth Complex Figure Test (RCFT) are widely used as a part of neuropsychological test batteries to assess cognitive function. Our objective was to confirm the prediction accuracies of the RCFT-copy and CDT for cognitive impairment $(\mathrm{Cl})$ using the convolutional neural network (CNN) algorithm as a screening tool.

Methods: The CDT and RCFT-copy data were obtained from patients aged 60 to 80 years who had more than 6 years of education. In total, 747 CDT and 980 RCFT-copy figures were utilized. CNN algorithms using TensorFlow (ver. 2.3.0) on the Colab cloud platform (www.colab.research.google.com) were used for preprocessing and modeling. We measured the prediction accuracy of each drawing test 10 times using this dataset: normal cognition (NC) vs. mild impairment of cognition (MI), NC vs. severely impaired cognition (SI), and $\mathrm{NC}$ vs. $\mathrm{Cl}(\mathrm{MI}+\mathrm{SI})$.

Results: The accuracy of the CDT was better for differentiating MI (CDT: 78.04 \pm 2.75, RCFT-copy: not being trained) and SI from NC (CDT: $91.45 \pm 0.83$, RCFT-copy: 90.27 \pm 1.52$)$; however, the RCFT-copy was better at predicting $\mathrm{Cl}(\mathrm{CDT}$ : $77.37 \pm 1.77, \mathrm{RCFT}$ : $83.52 \pm 1.41)$. The accuracy for 3-way classification (NC vs. MI vs. SI) was approximately $71 \%$ for both tests; no significant difference was found.

Conclusions: The two drawing tests showed good performance for predicting severe impairment of cognition; however, a drawing test alone is not enough to predict overall $\mathrm{Cl}$. There are some limitations to our study: the sample size was small, all participants did not perform both the CDT and RCFT-copy, and only the copy condition of the RCFT was used. Algorithms involving memory performance and longitudinal changes are worth future exploration. These results may contribute to improved home-based healthcare delivery.

\section{Background}

There is a growing interest in the introduction of artificial intelligence in clinical medicine (1-3). Efforts are underway to predict and diagnose prodromal or early-stage dementia (4-7) at home and in clinical settings $(8,9)$.

Dementia is a state of cognitive impairment $(\mathrm{Cl})$ including loss of memory, language, problem solving, and executive functions that is severe enough to impair daily life. Many studies have demonstrated the presence of mild cognitive impairment $(\mathrm{MCl})$, which is not severe enough to impair social and occupational activities, in the prodromal stage of dementia $(10,11)$. Neuropsychological testing is required in people with complaints of cognitive problems for an establishing objective diagnosis (12). Most of the screening tools have been constructed based on neuropsychological tests. The ReyOsterrieth Complex Figure Test (RCFT) is widely used by neuropsychologists to assess cognitive function. The test was first developed by Rey in 1941 (13) and has proved to be a useful tool for analyzing visuospatial construction, perceptual organization, and visual memory in clinical evaluations and research studies (14). Patients with parieto-occipital lesions, especially on the right side, have difficulties 
in spatial organization of the drawing, probably because of visual disorientation (15), while patients with frontal lobe damage show impairment in programming abilities in figure reproduction $(16,17)$. Earlystage Alzheimer's disease (AD) patients perform poorly on this test (18). Seo et al. showed that that the copy condition of the test was associated with spatial organization and planning and significantly predicted the conversion to pre- $\mathrm{MCl}$ or $\mathrm{MCl}$ (19). The salience of visuospatial and organizational skills as evaluated by the copy condition of the RCFT differed according to the level of intelligence (20). To obtain a more quantitative value for the accuracy of a participant's drawing, many researchers use the RCFT based on the Osterrieth scoring criteria to diagnose $\mathrm{Cl}(21)$.

The Clock Drawing Test (CDT) is also widely used as a screening test for dementia patients because of the following advantages: simple to use and reflection of a variety of cognitive functions, including visuospatial function, frontal lobe execution, and memory of clock concepts. The CDT requires the subject to draw the hour and minute hands of the clock to show the time "11:10." This can lead to a "selfstimulating error response," one of the errors in drawing the clock. It is necessary to suppress the tendency to draw a needle in a number called "10" driven by the perceptual level of information instead of "2" which is a meaningful number to indicate "10" (22). Studies related to dementia have reported that $\mathrm{CDT}$ is useful in screening of cognitive impairment $(23,24)$, and that is can be used for screening $\mathrm{MCl}$ [11]. The CDT has a variety of scoring systems (25). Among them, the Consortium to Establish a Registry for Alzheimer's Disease Clock Drawing Test (CERAD-CDT) (26) is known as the simplest method with high diagnostic efficiency (27).

Detection of the severity of dementia is important for clinical and research purposes, and the Clinical Dementia Rating Scale (CDR) is one of the most commonly used tools for assessment. The CDR comprises the global and sum of boxes (SOB) scores. The CDR-SOB is considered a more detailed quantitative index than the global score and provides more information regarding patients with mild dementia. Studies have shown that CDR-SOB scores may have the potential for discriminating between patients with $\mathrm{MCl}$ and patients with a very early stage of $A D$ dementia who are assigned a global $C D R$ score of 0.5 . Patients with $\mathrm{MCl}$ were assigned a CDR-SOB score of $1.8 \pm 0.8$, and very mild AD patients were assigned a CDR-SOB score of $3.0 \pm 0.8$ (28). O'Bryant et al. suggested the following staging system based on SOB scores: CDR-SOB 0, normal; 0.5-2.5, questionable impairment; and 3.0-4.0, very mild dementia (29). We also assessed the severity of dementia based on CDR-SOB score.

Several researchers have demonstrated that a digital CDT of limited number of subjects was able to differentiate patients with $A D$ and other dementia syndromes from normal controls using machine learning $(30,31)$. However, digital CDT needs special equipment, and in deep learning, more data of good quality can expect better results. Therefore, we predicted cognitive impairment with deep learning based on more drawing test data than previous study. Conventional CDT and RCFT are drawing tests that evaluate cognitive changes and are constituents of neuropsychological test batteries. We questioned whether these two simple drawing tests can be used as screening tests to predict $\mathrm{Cl}$ using convolutional neural network (CNN) algorithms. We also questioned whether the $\mathrm{CDT}$, which measures various cognitive 
functions, could be better in predicting $\mathrm{Cl}$ than the RCFT. Our objective was to evaluate the prediction accuracies of these two tests for $\mathrm{Cl}$ and to compare them.

\section{Methods}

\section{Dataset}

Anonymous neuropsychological data from Jan. 2018 to Sep. 2020 at the Memory Clinics at Seoul National University Bundang Hospital and Chung-Ang University Hospital were retrospectively collected. The RCFT-copy and CDT figures that were drawn by patients aged 60-80 years with more than 6 years of education were selected. There were a total of 747 CDT and 980 RCFT-copy figures.

The original RCFT (13) and CDT were conducted by trained psychologists in the neurology outpatient testing room. The participants were given an A4 size paper and a pencil and instructed to copy the "Rey complex figure" and/or draw a "clock" indicating the time "11:10." During the CDT, the following instructions were given: "You have to draw a clock. Draw a circle first and write all the numbers in it." After the patients wrote the numbers, they were instructed as follows: "Now draw hands on the clock to point 11:10." Test participants were clinically classified by dementia-specialized clinicians based on the CDRSOB score into the following groups: normal cognition (NC), $0-1.5$; mild impairment of cognition (MI), 2.0-3.5; and severe impairment of cognition (SI), 4 (29).

\section{Model training and statistical analyses}

The dataset of the CDT and RCFT-copy figures were organized into four classes: NC vs. MI, NC vs. SI, NC vs. $\mathrm{Cl}(\mathrm{MI}+\mathrm{SI})$, and NC vs. MI vs. SI. The datasets were prepared for three 2-way evaluations and one 3way evaluation with respect to each CDT and RCFT-copy figure. The 2-way classifications for differentiating $\mathrm{MI}, \mathrm{SI}$, or $\mathrm{Cl}$ from NC were performed in the CDT and RCFT datasets. The 3-way classification differentiated NC, MI, and SI in both drawing tests datasets.

All algorithms were performed on the Colab cloud platform (www.colab.research.google.com). To model the algorithm, the dataset was put through the following preprocessing steps. As the dataset was relatively small for machine learning, we augmented the image data. We made a replica image with $10 \%$ height reduction and a replica with $10 \%$ width reduction from each original drawing. All images including the original drawing and the two replicas were placed in a 600-dpi template.

TensorFlow (ver. 2.3.0) on Colab, which is a commonly used open-source software library for machine learning developed by Google based on Python, was used for preprocessing and modeling (32). As an example, the code that predict Cl with RCFT-copy and is given in the Supplementary Table. We imported the data in the ".png" format and used the "validation_split" function from "tf.keras.preprocessing.image_dataset_from_directory" to randomly split the data into training and test datasets. The training data size was $70 \%$, which indicated the percentage of the data to be withheld for training; the validation dataset was thus composed of the remaining $30 \%$ of the data. The features were 
normalized with "tensorflow.keras.layers.experimental.preprocession.Rescaling(1./255)." We implemented data augmentation with "RandomZoom" and "RandomRotation" using "experimental Keras Preprocessing Layers." This artificial neural network consists of five convolutional and maxpooling layers, and a dropout layer was inserted before connecting it to a fully connected neural network. The dropout rate was $0.2-0.3$; therefore, $2-3$ out of 10 weights were connected to the next layer to prevent overfitting. The cost was calculated using "Sparse_Categorical_Crossentropy" and minimized by means of the "adam" optimizer method. Model training was performed with a batch size of 20 and 40-72 epochs depending on the dataset. During model training, optimal dropout rates and epochs were found and adjusted. After the dropout rate and epochs were defined, we obtained the average prediction accuracy based on 10 trials.

\section{Results}

Demographic and clinical characteristics including age, education level, and Mini-Mental Status Examination scores of the CDT and RCFT-copy datasets are given in Table 1. No differences in age and education levels were found among the NC, MI, and SI groups in the CDT and RCFT-copy datasets ( $p$ > 0.05).

Table 1

Demographic and clinical characteristics according to cognition status based on the CDT and RCFT-copy

\begin{tabular}{|c|c|c|c|c|c|}
\hline & & $\mathrm{NC}$ & MI & SI & Total \\
\hline \multirow[t]{4}{*}{ CDT } & No. of subjects & $454(60.8 \%)$ & $179(24.0 \%)$ & $114(15.3 \%)$ & 747 \\
\hline & Age (years $\pm S D$ ) & $69.1 \pm 7.6$ & $70.5 \pm 7.7$ & $72.8 \pm 7.5$ & \\
\hline & Education (years $\pm S D$ ) & $11.6 \pm 3.2$ & $11.2 \pm 3.9$ & $10.5 \pm 4.2$ & \\
\hline & MMSE & $26.0 \pm 3.1$ & $24.4 \pm 2.3$ & $21.9 \pm 2.9$ & \\
\hline \multirow[t]{4}{*}{ RCFT-copy } & No. of subjects & $411(41.9 \%)$ & 367 (37.4\%) & $202(20.6 \%)$ & 980 \\
\hline & Age (years $\pm S D$ ) & $70.2 \pm 7.8$ & $70.2 \pm 7.2$ & $72.7 \pm 6.1$ & \\
\hline & Education (years $\pm S D$ ) & $11.9 \pm 3.5$ & $11.2 \pm 3.7$ & $10.5 \pm 3.4$ & \\
\hline & MMSE & $26.2 \pm 3.2$ & $24.5 \pm 2.4$ & $21.7 \pm 2.8$ & \\
\hline
\end{tabular}

The accuracy of the CDT was better for differentiating MI (CDT: $78.04 \pm 2.75$, RCFT: not being trained) and SI from NC (CDT: $91.45 \pm 0.83$, RCFT: $90.27 \pm 1.52$ ) (Table 2); however, the RCFT-copy was better at predicting $\mathrm{Cl}$ (CDT: $77.37 \pm 1.77, \mathrm{RCFT}: 83,52 \pm 1.41$ ). The accuracy of the 3-way classification (NC vs. MI vs. SI) was approximately $71 \%$ (Fig. 1). There was no significant difference between the two tests. 
Table 2

Mean accuracies for the prediction of cognitive impairment of the CDT and RCFT-copy

\begin{tabular}{|c|c|c|c|}
\hline & No. of images & $\begin{array}{l}\text { No. of training: validation } \\
\text { dataset }\end{array}$ & $\begin{array}{l}\text { Mean Accuracy of validation } \\
\text { dataset }\end{array}$ \\
\hline \multirow[t]{8}{*}{ CDT } & $\mathrm{NC}$ vs $\mathrm{Cl}$ & 1569:672 & $77.37 \pm 1.77$ \\
\hline & $(1362: 879)$ & & \\
\hline & NC vs MI & 1330:569 & $78.04 \pm 2.75$ \\
\hline & $(1362: 537)$ & & \\
\hline & NC vs SI & 1193:511 & $91.45 \pm 0.83$ \\
\hline & $(1362: 342)$ & & \\
\hline & $\mathrm{NC}$ vs $\mathrm{Ml}$ vs $\mathrm{SI}$ & 1569:672 & $71.06 \pm 0.75$ \\
\hline & $(1362: 537: 342)$ & & \\
\hline \multirow{8}{*}{$\begin{array}{l}\text { RCFT- } \\
\text { copy }\end{array}$} & $\mathrm{NC}$ vs $\mathrm{Cl}$ & $2058: 882$ & $83.52 \pm 1.41$ \\
\hline & $(1233: 1707))$ & & \\
\hline & $\mathrm{NC}$ vs Ml & $1634: 700$ & NT \\
\hline & $(1233: 1101)$ & & \\
\hline & NC vs SI & $1288: 551$ & $90.10 \pm 1.33$ \\
\hline & $(1233: 606)$ & & \\
\hline & NC vs MI vs SI & $2058: 882$ & $71.50 \pm 1.16$ \\
\hline & $(1233: 1101: 606)$ & & \\
\hline $\begin{array}{l}\text { CDT, } \\
\text { impa }\end{array}$ & $\begin{array}{l}\text { wing test; RCFT, } \\
\text { f cognition; SI, se }\end{array}$ & $\begin{array}{l}\text {-Osterrieth Complex Fig } \\
\text { e impairment of cognitior }\end{array}$ & $\begin{array}{l}\text {; NC, normal cognition; MI, mild } \\
\text { t being trained }\end{array}$ \\
\hline
\end{tabular}

\section{Discussion}

The algorithm for predicting $\mathrm{Cl}$ was more accurate in the RCFT-copy dataset than in the CDT dataset, but the algorithm for MI in the RCFT-copy training dataset could not be trained. However, at other levels of cognitive impairment, no significant difference was found between the two tests in predictive accuracy. We had believed that the CDT would be superior to the RCFT in predicting $\mathrm{Cl}$; this is because the RCFT simply evaluates visual constructional function based on copying of the figure, whereas the CDT not only evaluates visual constructional function and clock semantics but also evaluates the inhibitory function (22). The CDT was thought to be more advantageous for evaluating various aspects of cognitive function. However, there was no difference between the two tests in distinguishing MI and SI from NC in the 3-way classification. Rather, the RCFT was better at distinguishing $\mathrm{Cl}$ (including $\mathrm{MI}$ and $\mathrm{SI}$ ) from $\mathrm{NC}$ in the 2-way classification. A voxel-based morphometric study that evaluated the relationship between the 
RCFT and brain volume showed that the RCFT score and the right caudate nucleus volume were positively correlated. Therefore, in addition to visuospatial function evaluation, the RCFT may be used to evaluate frontal executive function (33). Another study found that poor copy scores in the RCFT were associated with greater beta amyloid burden in the frontal area on C-Pittsburgh B positron emission tomography/computed tomography (C-PiB-PET/CT) and F-FC119S PET/CT (34). These previous studies have shown that the RCFT is associated with cognitive functions other than visual constructional function.

More than $90 \%$ accuracy was seen for the prediction of SI in the validation dataset; however, the accuracy of differentiating between $\mathrm{MI}$ and $\mathrm{NC}$ was less than $80 \%$. Only a slight cognitive decline was difficult to detect using the CDT. Moreover, the RCFT-copy was not well trained in the training dataset, and thus, the validation dataset could not be evaluated.

There is a limitation in directly comparing the RCFT-copy and CDT machine learning algorithms as many patients had performed only one of the two drawing tests. Overall, it seems that there is no difference between the two algorithms in predicting $\mathrm{Cl}$. However, as the RCFT-copy algorithm could not be trained to select a patient with $\mathrm{MI}$, the CDT algorithm seemed advantageous.

Age and education have a strong effect on the performance in these tests $(35,36)$. Most of the available norms provide either percentile scores/means and standard deviations for age defined classes. However, in this study, age and educational levels were not included in the algorithm. We selected participants according to age and education levels. Their age ranged from 60-80 years, as changes in cognitive function are expected in this age range, and they had more than 6 years of formal education (to minimize the impact of low education on the performance in the drawing tests). If more substantial data could be obtained, it would be possible to predict $\mathrm{Cl}$ based on the variables of age and education level.

Although the two drawing tests alone cannot sufficiently predict $\mathrm{Cl}$ cross-sectionally, detecting changes in cognition using a longitudinal dataset is worth future exploration. The results from drawing-based neuropsychological tests and CNN algorithms can assist telemedicine or home-based care services.

\section{Limitations}

There are several limitations to our study. The small sample size may limit the generalizability of our findings. All subjects did not perform both the CDT and RCFT-copy, which can limit the direct comparison of the two tests. In this study, only the copy condition of the RCFT was used. The RCFT consists of copy and visual memory recall (immediate and delayed recall) conditions. Memory performance is important for the screening of $\mathrm{Cl}$. Therefore, including the delayed recall condition of the RCFT in future studies may help in better prediction of $\mathrm{Cl}$. The memory recall condition of the RCFT is thought to require a more complex machine learning model; this approach will be attempted in our future research.

\section{Conclusions}


The CDT and RCFT-copy showed good performance for predicting severe impairment of cognition; however, drawing tests alone are not enough to predict overall $\mathrm{Cl}$. Results from drawing tests and CNN algorithms can help improve home-based healthcare delivery. Algorithms involving memory performance and longitudinal changes are worth exploring in future studies.

\section{Abbreviations}

CDT: Clock drawing test

RCFT: Rey-Osterrieth Complex Figure Test

RCFT-copy: Rey-Osterrieth Complex Figure Test-copy

CNN: Convolutional neural network

MCl: Mild cognitive impairment

NC: Normal cognition

Ml: Mild impairment of cognition

Sl: Severe impairment of cognition

Cl: Cognitive impairment

CERAD-CDT: Consortium to Establish a Registry for Alzheimer's Disease Clock Drawing Test

CDR: Clinical Dementia Rating Scale

SOB: Sum of Boxes

\section{Declarations}

\section{Ethics approval and consent to participate}

Anonymous neuropsychological test datasets were used retrospectively from Seoul National University Bundang Hospital and Chung-Ang University Hospital. The study was approved by the institutional review boards of Seoul National University Bundang Hospital and Chung-Ang University Hospital (approval numbers, 1792-012-300). The need for written informed consent was waived due to the nature of the study.

\section{Consent for publication}

Not applicable. 
Availability of data and materials

The datasets used and/or analyzed during the current study are available from the corresponding author on reasonable request.

\section{Competing interests}

The authors declare that they have no competing interests.

\section{Funding}

The costs for manuscript publication, design of the study, data management, and writing of the manuscript were supported by the Ministry of Education of the Republic of Korea and the National Research Foundation of Korea (NRF-2017S1A6A3A01078538).

\section{Authors' contributions}

YCY planned, organized, and designed all experiments and results, collected data, and wrote the manuscript. JP, NR, and MJB collected data and reviewed and revised the manuscript. JJ, YHP, SA, HS, and KP reviewed and revised the manuscript. SYK reviewed all experiments and results, collected clinical data, and supervised the study. All authors read and approved the final version of the manuscript.

\section{References}

1. Benjamens S, Dhunnoo P, Mesko B. The state of artificial intelligence-based FDA-approved medical devices and algorithms: an online database. NPJ Digit Med. 2020;3:118.

2. Ramkumar PN, Kunze KN, Haeberle HS, Karnuta JM, Luu BC, Nwachukwu BU, et al. Clinical and Research Medical Applications of Artificial Intelligence. Arthroscopy. 2020.

3. Albahri OS, Zaidan AA, Albahri AS, Zaidan BB, Abdulkareem KH, Al-Qaysi ZT, et al. Systematic review of artificial intelligence techniques in the detection and classification of COVID-19 medical images in terms of evaluation and benchmarking: Taxonomy analysis, challenges, future solutions and methodological aspects. J Infect Public Health. 2020;13(10):1381-96.

4. Aschwanden D, Aichele S, Ghisletta P, Terracciano A, Kliegel M, Sutin AR, et al. Predicting Cognitive Impairment and Dementia: A Machine Learning Approach. J Alzheimers Dis. 2020;75(3):717-28.

5. Chen R, Herskovits EH. Machine-learning techniques for building a diagnostic model for very mild dementia. Neuroimage. 2010;52(1):234-44.

6. Dallora AL, Eivazzadeh S, Mendes E, Berglund J, Anderberg P. Machine learning and microsimulation techniques on the prognosis of dementia: A systematic literature review. PLoS One. 2017;12(6):e0179804.

7. Nori VS, Hane CA, Crown WH, Au R, Burke WJ, Sanghavi DM, et al. Machine learning models to predict onset of dementia: A label learning approach. Alzheimers Dement (N Y). 2019;5:918-25. 
8. Ahamed F, Shahrestani S, Cheung H. Internet of Things and Machine Learning for Healthy Ageing: Identifying the Early Signs of Dementia. Sensors (Basel). 2020;20(21).

9. Enshaeifar S, Zoha A, Markides A, Skillman S, Acton ST, Elsaleh T, et al. Health management and pattern analysis of daily living activities of people with dementia using in-home sensors and machine learning techniques. PLoS One. 2018;13(5):e0195605.

10. Breton A, Casey D, Arnaoutoglou NA. Cognitive tests for the detection of mild cognitive impairment $(\mathrm{MCl})$, the prodromal stage of dementia: Meta-analysis of diagnostic accuracy studies. Int $\mathrm{J}$ Geriatr Psychiatry. 2019;34(2):233-42.

11. Petersen RC, Smith GE, Waring SC, Ivnik RJ, Tangalos EG, Kokmen E. Mild cognitive impairment: clinical characterization and outcome. Arch Neurol. 1999;56(3):303-8.

12. Ahn HJ, Chin J, Park A, Lee BH, Suh MK, Seo SW, et al. Seoul Neuropsychological Screening Batterydementia version (SNSB-D): a useful tool for assessing and monitoring cognitive impairments in dementia patients. J Korean Med Sci. 2010;25(7):1071-6.

13. Rey A. L'examen psychologique dans les cas d'encéphalopathie traumatique. (Les problems.). [The psychological examination in cases of traumatic encepholopathy. Problems.]. Archives de Psychologie. 1941;28:215-85.

14. Cherrier MM, Mendez MF, Dave M, Perryman KM. Performance on the Rey-Osterrieth Complex Figure Test in Alzheimer disease and vascular dementia. Neuropsychiatry Neuropsychol Behav Neurol. 1999;12(2):95-101.

15. Binder LM. Constructional strategies on complex figure drawings after unilateral brain damage. J Clin Neuropsychol. 1982;4(1):51-8.

16. Pillon B. Troubles visuo-constructifs et méthodes de compensation: Résultats de 85 patients atteints de lésions cérébrales,. Neuropsychologia. 1981;19(3):375-83.

17. Lezak MD. Neuropsychological assessment. 3rd. ed. New York: Oxford; 1995.

18. Cummings JL, Benson DF. Dementia of the Alzheimer type. An inventory of diagnostic clinical features. J Am Geriatr Soc. 1986;34(1):12-9.

19. Seo EH, Kim H, Choi KY, Lee KH, Choo IH. Pre-Mild Cognitive Impairment: Can Visual Memory Predict Who Rapidly Convert to Mild Cognitive Impairment? Psychiatry Investigation. 2018;15(9):869-75.

20. Fujii DE, Lloyd HA, Miyamoto K. The salience of visuospatial and organizational skills in reproducing the Rey-Osterreith Complex Figure in subjects with high and low IQs. Clin Neuropsychol. 2000;14(4):551-4.

21. Rey A, Ferrara-Mori G, appliquée Cdp. Reattivo della figura complessa: Manuale: Organizzaziºni speciali; 1967.

22. Shulman Kl. Clock-drawing: is it the ideal cognitive screening test? International Journal of Geriatric Psychiatry. 2000;15(6):548-61.

23. Kim S, Jahng S, Yu K-H, Lee B-C, Kang Y. Usefulness of the Clock Drawing Test as a Cognitive Screening Instrument for Mild Cognitive Impairment and Mild Dementia: an Evaluation Using Three 
Scoring Systems. Dement Neurocogn Disord. 2018;17(3):100-9.

24. Allone C, Lo Buono V, Corallo F, Bonanno L, Palmeri R, Di Lorenzo G, et al. Cognitive impairment in Parkinson's disease, Alzheimer's dementia, and vascular dementia: the role of the clock-drawing test. Psychogeriatrics. 2018;18(2):123-31.

25. Lee KS, Kim EA, Hong CH, Lee DW, Oh BH, Cheong HK. Clock drawing test in mild cognitive impairment: quantitative analysis of four scoring methods and qualitative analysis. Dement Geriatr Cogn Disord. 2008;26(6):483-9.

26. Muller S, Herde L, Preische O, Zeller A, Heymann P, Robens S, et al. Diagnostic value of digital clock drawing test in comparison with CERAD neuropsychological battery total score for discrimination of patients in the early course of Alzheimer's disease from healthy individuals. Sci Rep. 2019;9(1):3543.

27. Lee KS, Cheong H-K, Oh BH, Hong CH, Lee D-W. Reliability and validity of four scoring methods of clock drawing test for screening dementia and mild cognitive impairment. Dementia and neurocognitive disorders. 2009:53.

28. Grundman M, Petersen RC, Ferris SH, Thomas RG, Aisen PS, Bennett DA, et al. Mild Cognitive Impairment Can Be Distinguished From Alzheimer Disease and Normal Aging for Clinical Trials. Archives of Neurology. 2004;61(1):59-66.

29. O’Bryant SE, Waring SC, Cullum CM, Hall J, Lacritz L, Massman PJ, et al. Staging Dementia Using Clinical Dementia Rating Scale Sum of Boxes Scores: A Texas Alzheimer's Research Consortium Study. Archives of Neurology. 2008;65(8):1091-5.

30. Davis R, Libon DJ, Au R, Pitman D, Penney DL. THink: Inferring Cognitive Status from Subtle Behaviors. Proc Conf AAAI Artif Intell. 2014;2014:2898-905.

31. Souillard-Mandar W, Davis R, Rudin C, Au R, Libon DJ, Swenson R, et al. Learning Classification Models of Cognitive Conditions from Subtle Behaviors in the Digital Clock Drawing Test. Mach Learn. 2016;102(3):393-441.

32. Rampasek L, Goldenberg A. TensorFlow: Biology's Gateway to Deep Learning? Cell Syst. 2016;2(1):12-4.

33. Lee S-Y, Yoon S-Y, Kim M-J, Rhee HY, Ryu C-W, Jahng G-H. Investigation of the Correlation between Seoul Neuropsychological Screening Battery Scores and the Gray Matter Volume after Correction of Covariates of the Age, Gender, and Genotypes in Patients with AD and MCl. J Korean Soc Magn Reson Med. 2013;17(4):294-307.

34. Park SY, Byun BH, Kim BI, Lim SM, Ko IO, Lee KC, et al. The correlation of neuropsychological evaluation with 11C-PiB and 18F-FC119S amyloid PET in mild cognitive impairment and Alzheimer disease. Medicine (Baltimore). 2020;99(16):e19620.

35. Rosselli M, Ardila A. Effects of age, education, and gender on the Rey-osterrieth complex figure. Clinical Neuropsychologist. 1991;5(4):370-6.

36. Boone KB, Lesser IM, Hill-gutierrez E, Berman NG, D'Elia LF. Rey-osterrieth complex figure performance in healthy, older adults: Relationship to age, education, sex, and IQ. Clinical Neuropsychologist. 1993;7(1):22-8. 
Figures

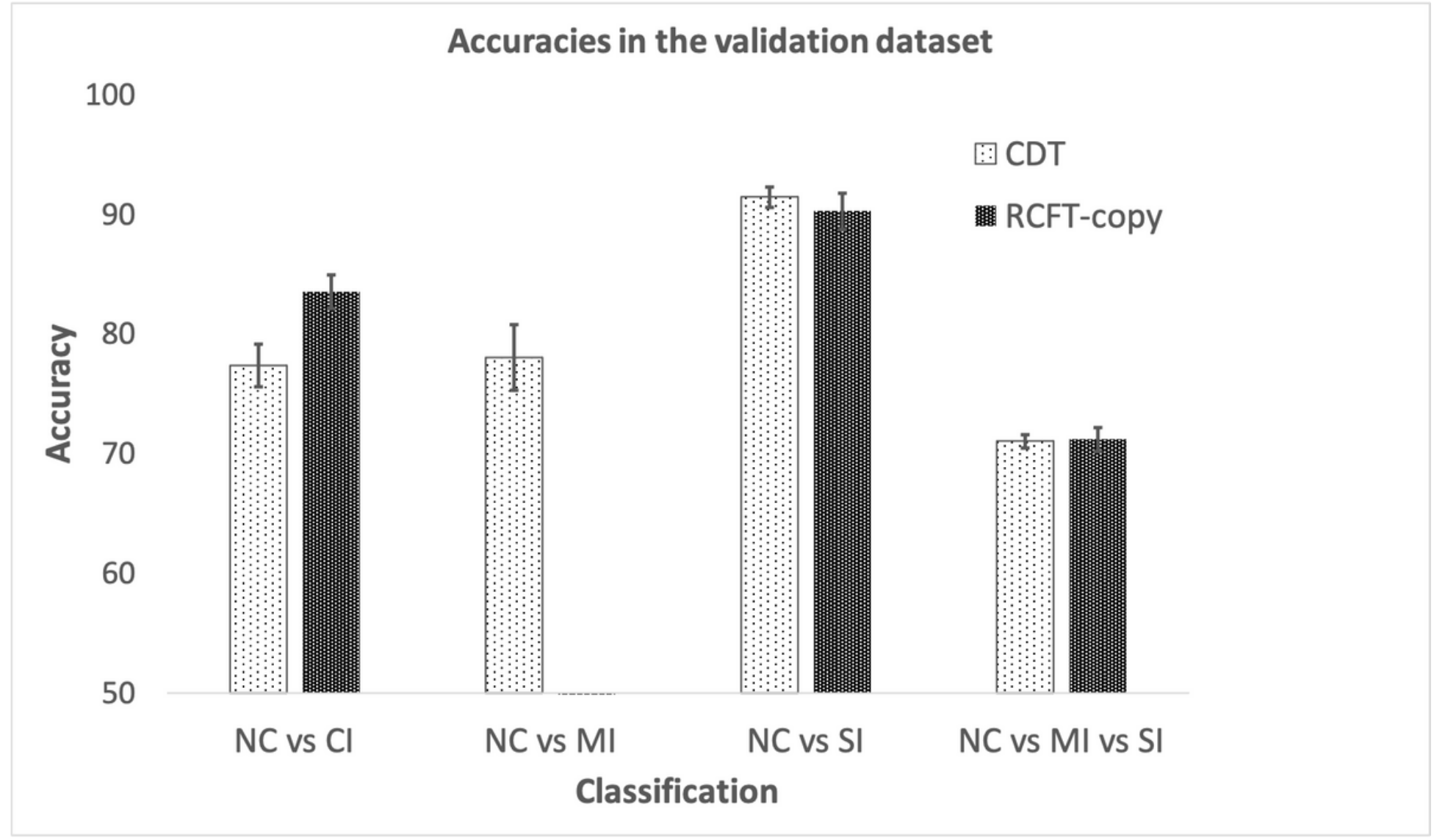

Figure 1

Accuracies of algorithms for the prediction of cognitive impairment in the validation dataset of the CDT and RCFT-copy.

\section{Supplementary Files}

This is a list of supplementary files associated with this preprint. Click to download.

- Supplementarytable1.docx 